\title{
Linear analysis of coupled lattices
}

\author{
D. Sagan and D. Rubin \\ Wilson Laboratory, Cornell University, Ithaca, New York 14853
}

(Received 8 February 1999; published 14 July 1999)

\begin{abstract}
A formalism for describing the coupled two-dimensional motion of high energy particle beams in a storage ring is developed and extended to circumstances where the coupling is very strong, such as for the Möbius twist accelerator.
\end{abstract}

PACS numbers: 29.20.Dh, 29.27.Bd

\section{INTRODUCTION}

The parametrization of linear one-dimensional optical systems using the so-called Twiss parameters, $\beta, \alpha$, and $\phi$, has been well established for some time [1]. The extension to two dimensions involves identifying the normal modes by transforming the full turn $4 \times 4$ transfer matrix to block diagonal form [2]. The system is then parametrized using the six Twiss parameters for both normal modes and four additional parameters specifying the orientation of the normal modes with respect to the laboratory $x, y$ axes.

For "weak" coupling where the normal modes correspond quite closely to uncoupled horizontal and vertical motion, this analysis is conceptually simple since, to first order in the coupling, the Twiss parameters are unaffected by the coupling. However, when the coupling is strong, the situation is more complicated since the Twiss parameters now no longer have their standard meanings. For example, $\sqrt{\beta}$ is now no longer proportional to the beam size. Furthermore, as discussed below, the identification of which mode is which at different places in the lattice can be obscured by the formalism. Since strong coupling has been put forward as a means to create round beams (for example, Talman [3] has proposed a "Möbius" twist accelerator), these subtleties need to be addressed, and this is the purpose of this paper.

\section{NORMAL MODE ANALYSIS}

\section{A. Similarity transformation}

Consider the motion of a particle in a storage ring and consider the 1-turn map $\mathbf{T}$ for the two-dimensional phase space $\mathbf{x}=\left(x, x^{\prime}, y, y^{\prime}\right)$. The motion is assumed linear so $\mathbf{T}$ can be represented as a $4 \times 4$ matrix. If we write $\mathbf{T}$ in terms of its $2 \times 2$ submatrices

$$
\mathbf{T}=\left(\begin{array}{cc}
\mathbf{M} & \mathbf{m} \\
\mathbf{n} & \mathbf{N}
\end{array}\right)
$$

then the motions in the $x$ and $y$ planes will be decoupled if $\mathbf{m}=\mathbf{n}=\mathbf{0}$. In this case, the standard Twiss analysis can be performed upon the matrices $\mathbf{M}$ and $\mathbf{N}$ [1]. If the motion is not decoupled then we seek a similarity transformation to normal modes form

$$
\mathbf{T}=\mathbf{V} \mathbf{U} \mathbf{V}^{-1},
$$

where $\mathbf{V}$ is symplectic, and $\mathbf{U}$ is of the form

$$
\mathbf{U}=\left(\begin{array}{ll}
\mathbf{A} & \mathbf{0} \\
\mathbf{0} & \mathbf{B}
\end{array}\right)
$$

There are many possible solutions for $\mathbf{V}$ that will satisfy the above conditions. Following Edwards and Teng [2], and Billing [4], $\mathbf{V}$ is written in the form

$$
\mathbf{V}=\left(\begin{array}{cc}
\gamma \mathbf{I} & \mathbf{C} \\
-\mathbf{C}^{+} & \gamma \mathbf{I}
\end{array}\right)
$$

where the symplectic conjugate is

$$
\mathbf{C}^{+}=\left(\begin{array}{cc}
C_{22} & -C_{12} \\
-C_{21} & C_{11}
\end{array}\right)
$$

Since we demand that $\mathbf{V}$ be symplectic, $\|\mathbf{V}\|=1$. With some algebra this condition relates $\gamma$ and $\mathbf{C}$ via

$$
\gamma^{2}+\|\mathbf{C}\|=1,
$$

and $\mathbf{V}^{-1}$ is given by

$$
\mathbf{V}^{-1}=\left(\begin{array}{cc}
\gamma \mathbf{I} & -\mathbf{C} \\
\mathbf{C}^{+} & \gamma \mathbf{I}
\end{array}\right)
$$

\section{B. Solution}

Substituting Eq. (4) into Eq. (2), and after some algebra, it is found that a solution for $\mathbf{V}$ is given by

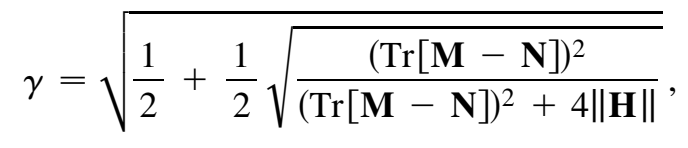

and

$$
\mathbf{C}=\frac{-\mathbf{H} \operatorname{sgn}(\operatorname{Tr}[\mathbf{M}-\mathbf{N}])}{\gamma \sqrt{(\operatorname{Tr}[\mathbf{M}-\mathbf{N}])^{2}+4\|\mathbf{H}\|}},
$$

where

$$
\mathbf{H} \equiv \mathbf{m}+\mathbf{n}^{+},
$$

and

$$
\operatorname{sgn} x= \begin{cases}-1, & \text { for } x \leq 0 \\ +1, & \text { for } x \geq 0\end{cases}
$$


Equations (8) and (9) give a real solution for Eq. (2) as long as

$$
(\operatorname{Tr}[\mathbf{M}-\mathbf{N}])^{2}+4\|\mathbf{H}\|>0 .
$$

However, Eq. (12) is also the condition for stable motion (see the discussion in Courant and Snyder [1] after Eq. 4.84). Thus, if the motion is stable, there is a solution to Eq. (2) with $\mathbf{V}$ of the form given by Eq. (4).

From the above equations the normal mode matrices $\mathbf{A}$ and $\mathbf{B}$ can be computed from Eqs. (1)-(4),

$$
\begin{aligned}
& \mathbf{A}=\gamma^{2} \mathbf{M}-\gamma\left(\mathbf{C n}+\mathbf{m} \mathbf{C}^{+}\right)+\mathbf{C N C}^{2}, \\
& \mathbf{B}=\gamma^{2} \mathbf{N}-\gamma\left(\mathbf{n C}+\mathbf{C}^{+} \mathbf{m}\right)+\mathbf{C M C}^{2} .
\end{aligned}
$$

From $\mathbf{A}$ and $\mathbf{B}$ the normal mode Twiss parameters can be computed using the standard formulas [5]

$$
\mathbf{A}=\left(\begin{array}{cc}
\cos \theta_{a}+\alpha_{a} \sin \theta_{a} & \beta_{a} \sin \theta_{a} \\
-\gamma_{a} \sin \theta_{a} & \cos \theta_{a}-\alpha_{a} \sin \theta_{a}
\end{array}\right),
$$

with a similar equation for the $b$ mode Twiss parameters. From Eq. (2), the normal mode coordinates $\mathbf{a}=$ $\left(a, a^{\prime}, b, b^{\prime}\right)$ are related to the laboratory frame via

$$
\mathbf{a}=\mathbf{V}^{-1} \mathbf{x} \text {. }
$$

It is important to remember here that since the normal mode axes are linear combinations of the laboratory axes, some standard equations used in analyzing onedimensional motion are not valid in the two-dimensional case. For example, the equation $2 \alpha=-d \beta / d s$ is now no longer valid.

\section{Second solution}

As long as $\|\mathbf{H}\|>0$ it is possible to construct a second solution for $\mathbf{V}$ which is given by

$$
\begin{gathered}
\gamma=\sqrt{\frac{1}{2}-\frac{1}{2} \sqrt{\frac{(\operatorname{Tr}[\mathbf{M}-\mathbf{N}])^{2}}{(\operatorname{Tr}[\mathbf{M}-\mathbf{N}])^{2}+4\|\mathbf{H}\|}},} \\
\mathbf{C}=\frac{\mathbf{H} \operatorname{sgn}(\operatorname{Tr}[\mathbf{M}-\mathbf{N}])}{\gamma \sqrt{(\operatorname{Tr}[\mathbf{M}-\mathbf{N}])^{2}+4\|\mathbf{H}\|}},
\end{gathered}
$$

Physically, the two solutions can be explained as follows: Call the two eigenmodes of the system " $h$ " and " $v$ " (for a physical picture think, for example, of the $h$ eigenmode as the mode with the higher tune and the $v$ mode as the mode with the lower tune). The difference between the two solutions is whether the $h$ mode is associated with the $a$ mode (i.e., gets placed in the upper-left-hand corner of the $\mathbf{U}$ matrix) or whether the $h$ mode is associated with the $b$ mode. From a physics standpoint it does not matter whether the $h$ mode is associated with the $a$ mode or the $b$ mode, but using a different $\gamma$ and $\mathbf{C}$ will change the normal mode matrices A and $\mathbf{B}$ [Eqs. (13) and (14)], and hence the computed values of the Twiss parameters will be different [see Eq. (15)]. Another way of looking at this is to note that even with one-dimensional motion the Twiss parameters are dependent upon the choice of axes. For example, a transformation

$$
\left(\begin{array}{c}
a \\
a^{\prime}
\end{array}\right)=\mathbf{G}_{x}\left(\begin{array}{c}
x \\
x^{\prime}
\end{array}\right)
$$

with $\mathbf{G}_{x}$ taken to be

$$
\mathbf{G}_{x}=\left(\begin{array}{cc}
\frac{1}{\sqrt{\beta_{x}}} & 0 \\
\frac{\alpha_{x}}{\sqrt{\beta_{x}}} & \sqrt{\beta_{x}}
\end{array}\right),
$$

will give, in terms of the new coordinates, normalized Twiss parameters of $\beta_{a}=1$ and $\alpha_{a}=0$. Thus, by using Eqs. (17) and (18) instead of Eqs. (8) and (9) the eigenaxes are changed [Eq. (16)] and this affects the values for the Twiss parameters.

For a weakly coupled lattice where $\mathbf{H}$ is "small," one should always choose the solution given by Eqs. (8) and (9) over Eqs. (17) and (18). There are two reasons for this. First, Eqs. (17) and (18) are not valid where $\|\mathbf{H}\|<$ 0. Second, with Eqs. (8) and (9), the interpretation of the similarity transformation is simple: $\mathbf{V}$ is nearly the identity matrix and the $a$ and $b$ modes are associated with the (nearly) horizontal and vertical modes, respectively.

For a highly coupled lattice, the situation becomes more complicated. Since there is only one solution where $\|\mathbf{H}\|<0$, it can happen that at one place in the ring where $\|\mathbf{H}\|<0$, the $h$ mode is forced to be associated with the $a$ mode and that, at another place in the ring, the $h$ mode is forced to be associated with the $b$ mode. Indeed, this characteristic has been found in investigations of Möbiustype lattices at the Cornell CESR ring. The switching of the $h$ and $v$ modes between the $a$ and $b$ modes as one propagates the Twiss parameters through the ring is something we call "mode flipping."

From the above discussion it is seen that the bookkeeping for a highly coupled lattice is more complicated than if the lattice is only slightly coupled. For example, the Twiss parameters at a point computed from similar lattices may be wildly different due to mode flipping. In other words, in order to compare two lattices to see how similar they are, one cannot just compare the Twiss parameters, but has to look in detail at what the eigenaxes are. This problem with identifying which mode is which at different points in the lattice is not due just to the choice of form for $\mathbf{V}$ [Eq. (4)]; it is inherent in the similarity transformation itself [Eq. (2)].

\section{NORMAL MODE PROPAGATION}

Given that the normal mode analysis has been done at point 1, and given the transfer matrix $\mathbf{T}_{12}$ between points 1 and 2, how can the normal mode analysis be propagated from 1 to 2 ? One straightforward way is simply to form the 1-turn matrix at point 2 ,

$$
\mathbf{T}_{2}=\mathbf{T}_{12} \mathbf{T}_{1} \mathbf{T}_{12}^{-1},
$$


and the analysis can be done as outlined in the previous section. Mode flips can be checked for by comparing the trace of the eigenmatrices, $\mathbf{A}$ and $\mathbf{B}$, between the two points. An alternative is to directly construct the transformation matrix between the eigenmatrices at the two points. A benefit with this method is that only $2 \times 2$ (rather than $4 \times 4$ ) matrices need to be used, which makes the calculation computationally quicker.

Using Eqs. (1) and (21), the propagation of the normal mode matrix $\mathbf{U}$ is given by

$$
\mathbf{U}_{2}=\mathbf{V}_{2}^{-1} \mathbf{T}_{2} \mathbf{V}_{2}=\mathbf{W}_{12} \mathbf{U}_{1} \mathbf{W}_{12}^{-1},
$$

where

$$
\mathbf{W}_{12} \equiv \mathbf{V}_{2}^{-1} \mathbf{T}_{12} \mathbf{V}_{1}
$$

$\mathbf{W}_{12}$ is the similarity transformation connecting the eigenmode matrices $\mathbf{U}_{1}$ and $\mathbf{U}_{2}$. Since the eigenmodes are independent there cannot be any terms in $\mathbf{W}_{12}$ that connect the two modes. Since the $\mathbf{U}_{i}$ are block diagonal, this means that $\mathbf{W}_{12}$ is either block diagonal or is "off-block diagonal" (has zeros on the $2 \times 2$ block diagonals). A formal proof of this is given in Appendix A. The exceptions to the above statement come when the modes can "mix" at the coupling resonance or at the stop band resonance. We will not consider these exceptional cases further. $\mathbf{W}_{12}$ will be off-block diagonal when there is a mode flip with the $a$ mode at point 1 becoming the $b$ mode at point 2 , and vice versa for the other mode.

\section{A. Propagation without a mode flip}

Consider first the case where $\mathbf{W}_{12}$ is block diagonal (no mode flip). In this case $\mathbf{W}_{12}$ has the form

$$
\mathbf{W}_{12}=\left(\begin{array}{cc}
\mathbf{E}_{12} & \mathbf{0} \\
\mathbf{0} & \mathbf{F}_{12}
\end{array}\right) \text {. }
$$

In terms of its $2 \times 2$ submatrices, $\mathbf{T}_{12}$ is written as

$$
\mathbf{T}_{12}=\left(\begin{array}{ll}
\mathbf{M}_{12} & \mathbf{m}_{12} \\
\mathbf{n}_{12} & \mathbf{N}_{12}
\end{array}\right) \text {. }
$$

From Eq. (23) we have $\mathbf{V}_{2} \mathbf{W}_{12}=\mathbf{T}_{12} \mathbf{V}_{1}$ which gives with Eqs. (24) and (25)

$$
\left(\begin{array}{cc}
\gamma_{2} \mathbf{E}_{12} & \mathbf{C}_{2} \mathbf{F}_{12} \\
-\mathbf{C}_{2}^{+} \mathbf{E}_{12} & \gamma_{2} \mathbf{F}_{12}
\end{array}\right)=\left(\begin{array}{cc}
\gamma_{1} \mathbf{M}_{12}-\mathbf{m}_{12} \mathbf{C}_{1}^{+} & \mathbf{M}_{12} \mathbf{C}_{1}+\gamma \mathbf{m}_{12} \\
\gamma_{1} \mathbf{n}_{12}-\mathbf{N}_{12} \mathbf{C}_{1}^{+} & \mathbf{n}_{12} \mathbf{C}_{1}+\gamma_{1} \mathbf{N}_{12}
\end{array}\right)
$$

Consider first the $(2,2)$ component in Eq. (26). Since $\mathbf{W}_{12}$ is the product of symplectic matrices, $\mathbf{W}_{12}$ is symplectic and thus $\left\|\mathbf{F}_{12}\right\|=1$. Hence

$$
\gamma_{2}^{2}=\left\|\mathbf{n}_{12} \mathbf{C}_{1}+\gamma_{1} \mathbf{N}_{12}\right\| .
$$

Equating the rest of the terms in Eq. (26) gives

$$
\begin{gathered}
\mathbf{E}_{12}=\left(\gamma_{1} \mathbf{M}_{12}-\mathbf{m}_{12} \mathbf{C}_{1}^{+}\right) / \gamma_{2}, \\
\mathbf{F}_{12}=\left(\mathbf{n}_{12} \mathbf{C}_{1}+\gamma_{1} \mathbf{N}_{12}\right) / \gamma_{2}, \\
\mathbf{C}_{2}=\left(\mathbf{M}_{12} \mathbf{C}_{1}+\gamma_{1} \mathbf{m}_{12}\right) \mathbf{F}_{12}^{-1} .
\end{gathered}
$$

From Eq. (24) the normal mode vectors propagate as

$$
\begin{aligned}
& \mathbf{a}_{2}=\mathbf{E}_{12} \mathbf{a}_{1}, \\
& \mathbf{b}_{2}=\mathbf{F}_{12} \mathbf{b}_{1} .
\end{aligned}
$$

In the special case of propagation through an element that does not couple $x$ and $y$ motions, $\mathbf{T}_{12}$ is block diagonal. That is, $\mathbf{n}_{12}=\mathbf{m}_{12}=\mathbf{0}$. In this case, $\mathbf{W}_{12}$ is automatically block diagonal and Eqs. (27) and (29) reduce to

$$
\begin{gathered}
\gamma_{2}=\gamma_{1}, \\
\mathbf{W}_{12}=\mathbf{T}_{12}, \\
\mathbf{C}_{2}=\mathbf{M}_{12} \mathbf{C}_{1} \mathbf{N}_{12}^{-1} .
\end{gathered}
$$

\section{B. Propagation with a mode flip}

For the case when $\mathbf{W}_{12}$ is off-block diagonal, so that $\mathbf{W}_{12}$ is of the form

$$
\mathbf{W}_{12}=\left(\begin{array}{cc}
0 & \mathbf{F}_{12} \\
\mathbf{E}_{12} & 0
\end{array}\right),
$$

an analysis analogous to that of the preceding section gives

$$
\gamma_{2}^{2}=\left\|\mathbf{M}_{12} \mathbf{C}_{1}+\gamma_{1} \mathbf{m}_{12}\right\|
$$

with

$$
\begin{aligned}
& \mathbf{E}_{12}=\left(\gamma_{1} \mathbf{n}_{12}-\mathbf{N}_{12} \mathbf{C}_{1}^{+}\right) / \gamma_{2}, \\
& \mathbf{F}_{12}=\left(\mathbf{M}_{12} \mathbf{C}_{1}+\gamma_{1} \mathbf{m}_{12}\right) / \gamma_{2}, \\
& \mathbf{C}_{2}=\left(\gamma_{1} \mathbf{M}_{12}-\mathbf{m}_{12} \mathbf{C}_{1}^{+}\right) \mathbf{E}_{12}^{-1},
\end{aligned}
$$

and the normal mode vectors propagate as

$$
\begin{aligned}
& \mathbf{b}_{2}=\mathbf{E}_{12} \mathbf{a}_{1}, \\
& \mathbf{a}_{2}=\mathbf{F}_{12} \mathbf{b}_{1} .
\end{aligned}
$$

Notice that if the right-hand side of Eq. (27) is zero or negative, then one is forced to mode flip and use the solution given by Eqs. (37)-(40). If $\gamma$ is calculated as a continuous function of longitudinal position, then the point at which a mode flip is forced is where $\gamma \rightarrow 0$. At such a place, the Twiss parameters and eigenaxes will be discontinuous. Furthermore, the integer part of the phase is not well defined across a mode flip. Note that since $\gamma$ is constant when propagated through elements without coupling [Eq. (34)], mode flipping will be forced only when propagating through an element that couples the $x-y$ motions. In any case, a lattice should always have an even number of mode flips so that the eigenaxes at the start and 
end of the full turn are the same. If this is not done, then the computed full turn phase advance will not be correct.

While it is not a necessary condition, the beta function as $\gamma \rightarrow 0$ may diverge to infinity and it is a fairly simple matter to construct examples that exhibit this behavior. Though $\beta$ may diverge, any physically meaningful parameter, for example the beam sizes, will remain finite (see below).

\section{IV. $\bar{C}$ COUPLING MATRIX}

The $2 \times 2$ submatrix $\mathbf{C}$ is a measure of the coupling: If $\mathbf{C}=\mathbf{0}$, then $\mathbf{V}=\mathbf{1}$ and the motion is decoupled. It turns out that it is convenient to normalize out the $\beta$ dependence in $\mathbf{C}$ via the matrix $\mathbf{G}$

$$
\mathbf{G} \equiv\left(\begin{array}{cc}
\mathbf{G}_{a} & \mathbf{0} \\
\mathbf{0} & \mathbf{G}_{b}
\end{array}\right),
$$

where $\mathbf{G}_{a}$ and $\mathbf{G}_{b}$ are the normalization matrices for the $a$ and $b$ modes, respectively, and have the form as given in Eq. (20). The normalized normal mode matrix $\overline{\mathbf{U}}$ is defined by

$$
\overline{\mathbf{U}}=\mathbf{G U G}^{-1},
$$

Using Eqs. (3), (15), (20), and (43) in Eq. (44) shows that $\overline{\mathbf{U}}$ is simply made up of rotation matrices.

$$
\overline{\mathbf{U}}=\left(\begin{array}{cc}
\mathbf{R}\left(\theta_{a}\right) & \mathbf{0} \\
\mathbf{0} & \mathbf{R}\left(\theta_{b}\right)
\end{array}\right),
$$

where the rotation matrix $\mathbf{R}$ is

$$
\mathbf{R}(\theta) \equiv\left(\begin{array}{cc}
\cos \theta & \sin \theta \\
-\sin \theta & \cos \theta
\end{array}\right) .
$$

The similarity transformation is now written in terms of $\overline{\mathbf{U}}$. Putting Eq. (44) into Eq. (2) gives

$$
\mathbf{T}=\mathbf{G}^{-1} \overline{\mathbf{V}} \overline{\mathbf{U}} \overline{\mathbf{V}}^{-1} \mathbf{G},
$$

where

$$
\overline{\mathbf{V}}=\mathbf{G V G}^{-1} \text {. }
$$

Using Eq. (43), $\overline{\mathbf{V}}$ can be written in the form

$$
\overline{\mathbf{V}}=\left(\begin{array}{cc}
\gamma \mathbf{I} & \overline{\mathbf{C}} \\
-\overline{\mathbf{C}}^{+} & \gamma \mathbf{I}
\end{array}\right),
$$

with the normalized matrix $\overline{\mathbf{C}}$ given by

$$
\overline{\mathbf{C}}=\mathbf{G}_{a} \mathbf{C G}_{b}^{-1} .
$$

To see how $\overline{\mathbf{C}}$ can be used, consider the motion when a single mode is excited [6]. The motion in normal mode space $\overline{\mathbf{a}}=\left(\bar{a}, \bar{a}^{\prime}, \bar{b}, \bar{b}^{\prime}\right)$ is particularly simple. For the $\bar{a}$ mode

$$
\left(\begin{array}{c}
\bar{a} \\
\bar{a}^{\prime}
\end{array}\right)=A_{a}\left(\begin{array}{c}
\cos \psi_{a} \\
\sin \psi_{a}
\end{array}\right)
$$

where the phase $\psi_{a}$ on the $n$th turn at position $s$ is

$$
\psi_{a}(n, s)=\theta_{a} n+\phi_{a}(s)+\phi_{a 0},
$$

where $\phi_{a}(s)$ is the betatron phase at point $s$, and $\phi_{a 0}$ is calculated from the initial conditions. Form Eq. (47) the transformation from normalized normal mode space to the laboratory frame is

$$
\mathbf{x}=\mathbf{G}^{-1} \overline{\mathbf{V}} \overline{\mathbf{a}} .
$$

Using this, the motion in the $x-y$ plane is

$$
\left(\begin{array}{l}
x \\
y
\end{array}\right)=A_{a}\left(\begin{array}{c}
\gamma \sqrt{\beta_{a}} \cos \psi_{a} \\
-\sqrt{\beta_{b}}\left(\bar{C}_{22} \cos \psi_{a}+\bar{C}_{12} \sin \psi_{a}\right)
\end{array}\right) .
$$

For the $b$ mode, a similar analysis can be done with the result

$$
\left(\begin{array}{l}
x \\
y
\end{array}\right)=A_{b}\left(\begin{array}{c}
\sqrt{\beta_{a}}\left(\bar{C}_{11} \cos \psi_{b}-\bar{C}_{12} \sin \psi_{b}\right) \\
\gamma \sqrt{\beta_{b}} \cos \psi_{b}
\end{array}\right) .
$$

$\overline{\mathbf{C}}$ is useful for characterizing the amount of coupling in a lattice [7]. Using modeling programs, measurements of $\overline{\mathbf{C}}$ also allow correction of the coupling [6]. From the above equations the rms beam sizes in the $x$ and $y$ planes for the $a$ mode are

$$
\sigma_{x, a}=\gamma \sqrt{\epsilon_{a} \beta_{a}},
$$

and

$$
\sigma_{y, a}=\sqrt{\epsilon_{a} \beta_{b}}\left[\bar{C}_{22}^{2}+\bar{C}_{12}^{2}\right]^{1 / 2},
$$

where $\epsilon_{a}$ is the $a$ mode emittance (which must be calculated using a formula generalized from the formula used in the one-dimensional case). For the $b$ mode

$$
\sigma_{x, b}=\sqrt{\epsilon_{b} \beta_{a}}\left[\bar{C}_{11}^{2}+\bar{C}_{12}^{2}\right]^{1 / 2},
$$

and

$$
\sigma_{y, b}=\gamma \sqrt{\epsilon_{b} \beta_{b}} .
$$

Since the normal mode motions are at different frequencies, the rms beam sizes add in quadrature. The total beam size in the $x$ and $y$ planes is then

$$
\sigma^{2}=\sigma_{a}^{2}+\sigma_{b}^{2}
$$

\section{ACKNOWLEDGMENTS}

The authors thank Richard Talman for helpful discussions and the rest of the CESR operations group for their support. This work was supported by the National Science Foundation.

\section{APPENDIX A: BLOCK DIAGONAL PROOF}

To show that $\mathbf{W}_{12}$ is block diagonal or off-block diagonal, we use the normalized $\overline{\mathbf{U}}$ [Eq. (44)]

$$
\overline{\mathbf{W}}_{12}=\overline{\mathbf{U}}_{2} \overline{\mathbf{W}}_{12} \overline{\mathbf{U}}_{1}^{-1}
$$

where

$$
\overline{\mathbf{W}}_{12}=\mathbf{G}_{2}^{-1} \mathbf{W}_{12} \mathbf{G}_{1},
$$

with $\mathbf{G}_{1}$ and $\mathbf{G}_{2}$ given by Eq. (43). Obviously $\mathbf{W}_{12}$ is block diagonal (or off-block diagonal) if and only if $\overline{\mathbf{W}}_{12}$ is. 
$\mathbf{U}_{1}$ will be of the form Eq. (45), and either $\mathbf{U}_{2}$ will also be of the same form or, if there has been a mode flip in between, will have $\mathbf{R}\left(\theta_{a}\right)$ and $\mathbf{R}\left(\theta_{b}\right)$ interchanged. We first take the nonmode flip case where the eigenmodes stay in their respective places between points 1 and 2. Writing

$$
\overline{\mathbf{W}}_{12}=\left(\begin{array}{ll}
\mathbf{p} & \mathbf{q} \\
\mathbf{r} & \mathbf{s}
\end{array}\right)
$$

and inserting this into Eq. (A1) gives for $\mathbf{q}$

$$
\mathbf{q}=\mathbf{R}\left(\theta_{a}\right) \mathbf{q} \mathbf{R}^{-1}\left(\theta_{b}\right) .
$$

We want to show that $\mathbf{q}=0$. To see this we note the following without proof:

(i) Any $2 \times 2$ matrix $\mathbf{z}$ can be decomposed into "rotational" and "antirotational" matrices

$$
\begin{aligned}
\mathbf{z}= & \left(\begin{array}{ll}
z_{11} & z_{12} \\
z_{21} & z_{22}
\end{array}\right) \\
= & \frac{1}{2}\left(\begin{array}{cc}
z_{11}-z_{22} & z_{12}+z_{21} \\
z_{12}+z_{21} & -\left(z_{11}-z_{22}\right)
\end{array}\right) \\
& +\frac{1}{2}\left(\begin{array}{cc}
z_{11}+z_{22} & z_{12}-z_{21} \\
-\left(z_{12}-z_{21}\right) & z_{11}+z_{22}
\end{array}\right) \\
= & \lambda \mathbf{S}(\phi)+\kappa \mathbf{R}(\theta),
\end{aligned}
$$

where $\lambda$ and $\kappa$ are constants, $\mathbf{R}$ is a rotational matrix given by Eq. (46), and $\mathbf{S}$ is of the form

$$
\mathbf{S}(\phi) \equiv\left(\begin{array}{cc}
\cos \phi & \sin \phi \\
\sin \phi & -\cos \phi
\end{array}\right)
$$

The inverse (if it exists) is

$$
\mathbf{z}^{-1}=\frac{1}{\lambda^{2}-\kappa^{2}}[\lambda \mathbf{S}(\phi)-\kappa \mathbf{R}(-\theta)] .
$$

(ii) Given any $\mathbf{z}$, the product $\lambda \mathbf{S}(\phi)$ is unique and the product $\kappa \mathbf{R}(\theta)$ is also unique so that if

$$
\begin{aligned}
\mathbf{z}_{1} & =\lambda_{1} \mathbf{S}\left(\phi_{1}\right)+\kappa_{1} \mathbf{R}\left(\theta_{1}\right) \\
\text { and } \quad \mathbf{z}_{2} & =\lambda_{2} \mathbf{S}\left(\phi_{2}\right)+\kappa_{2} \mathbf{R}\left(\theta_{2}\right),
\end{aligned}
$$

then $\mathbf{z}_{1}=\mathbf{z}_{2}$ if and only if

$$
\lambda_{1} \mathbf{S}\left(\phi_{1}\right)=\lambda_{2} \mathbf{S}\left(\phi_{2}\right),
$$

and

$$
\kappa_{1} \mathbf{R}\left(\theta_{1}\right)=\kappa_{2} \mathbf{R}\left(\theta_{2}\right) .
$$

(iii) Rotation matrices and antirotation matrices multiply as

$$
\begin{aligned}
& \mathbf{R}\left(\theta_{1}\right) \mathbf{R}\left(\theta_{2}\right)=\mathbf{R}\left(\theta_{1}+\theta_{2}\right), \\
& \mathbf{S}\left(\phi_{1}\right) \mathbf{R}\left(\theta_{2}\right)=\mathbf{S}\left(\phi_{1}+\theta_{2}\right), \\
& \mathbf{R}\left(\theta_{2}\right) \mathbf{S}\left(\phi_{1}\right)=\mathbf{S}\left(\phi_{1}-\theta_{2}\right), \\
& \mathbf{S}\left(\phi_{1}\right) \mathbf{S}\left(\phi_{2}\right)=\mathbf{R}\left(\phi_{2}-\phi_{1}\right) .
\end{aligned}
$$

Using the above, $\mathbf{q}$ is written in the form $\mathbf{q}=$ $\lambda \mathbf{S}(\phi)+\kappa \mathbf{R}(\theta)$. Using this with assertions (ii) and (iii) in Eq. (A4) gives

$$
\begin{aligned}
\lambda \mathbf{S}(\phi) & =\lambda \mathbf{S}\left(\phi-\theta_{a}-\theta_{b}\right), \\
\kappa \mathbf{R}(\theta) & =\kappa \mathbf{R}\left(\theta+\theta_{a}-\theta_{b}\right) .
\end{aligned}
$$

Since we are assuming that we are not at the stop band resonance where $\theta_{a}+\theta_{b}=2 \pi m$ for some integer $m$, Eq. (A12) can be true only if $\lambda=0$. Additionally, since we are assuming that we are not at the coupling resonance where $\theta_{a}-\theta_{b}=2 \pi n$ for some integer $n$, Eq. (A13) can be true only if $\kappa=0$. Thus $\mathbf{q}=\mathbf{0}$. Similarly, it can be shown that $\mathbf{r}=\mathbf{0}$, and thus $\overline{\mathbf{W}}_{12}$ and $\mathbf{W}_{12}$ are block diagonal. For the case where there is a mode flip, then it can similarly be shown that $\mathbf{p}=\mathbf{s}=\mathbf{0}$, and hence $\mathbf{W}_{12}$ is off-block diagonal.

\section{APPENDIX B: MODE FLIP TRANSFORMATION}

Given one decomposition solution for $\mathbf{V}$ [Eqs. (8) and (9), or Eqs. (17) and (18)] with $\gamma<1$, the other "flipped" solution (denoted by a subscript $f$ ) is related to the first via

$$
\begin{gathered}
\gamma_{f}=\sqrt{1-\gamma^{2}}, \quad \mathbf{C}_{f}=\frac{-\gamma}{\gamma_{f}} \mathbf{C}, \\
\mathbf{U}_{f}=\mathbf{W U W}^{-1},
\end{gathered}
$$

where

$$
\mathbf{W}=\left(\begin{array}{cc}
\mathbf{0} & \frac{\mathbf{C}}{\gamma_{f}} \\
\frac{-\mathbf{C}^{+}}{\gamma_{f}} & \mathbf{0}
\end{array}\right),
$$

which gives for the

$$
\mathbf{A}_{f}=\frac{1}{\gamma_{f}^{2}} \mathbf{C B C}^{+}, \quad \mathbf{B}_{f}=\frac{1}{\gamma_{f}^{2}} \mathbf{C}^{+} \mathbf{A C} .
$$

[1] E. D. Courant and H.S. Snyder, Ann. Phys. (Paris) 3, 1-48 (1958).

[2] D. Edwards and L. Teng, IEEE Trans. Nucl. Sci. 20, 3 (1973).

[3] R. Talman, Phys. Rev. Lett. 74, 1590 (1995).

[4] M. Billing, Cornell Report No. CBN 85-2, 1985.

[5] C. Bovet, R. Gouiran, I. Gumowski, and K.H. Reich, CERN Report No. CERN/MPS-SI/Int. DL/70/4, 1970.

[6] P. Bagley and D. Rubin, in Proceedings of the 1989 Particle Accelerator Conference, Chicago, Illinois (IEEE, Piscataway, NJ, 1989), p. 874.

[7] D. Sagan, in Proceedings of the 1995 Particle Accelerator Conference, Dallas, Texas (IEEE, Piscataway, NJ, 1996), p. 3382 . 